\title{
The AGB-star Phenomenon: Setting the Stage
}

\author{
Hans Olofsson \\ Stockholm Observatory, SE-133 36 Saltsjöbaden, Sweden
}

\begin{abstract}
The asymptotic giant branch (AGB) evolution of stars is interesting from many points of view. It is the final evolutionary phase for the large majority of all stars in the Universe that have left the main sequence. During this stage they contribute to the chemical evolution of galaxies, contribute to the integrated starlight of many galaxies, may provide the only interstellar matter there is in some galaxies, and can, due to their high luminosities and ages, be used as important probes of galactic structure and dynamics. It is also the final evolutionary stage of our own star, the Sun, and hence touches on aspects beyond the immediate astronomical interest. Furthermore, these stars provide us with fascinating systems, where intricate interplays between physical and chemical processes take place, that we simply would like to understand. In this introductory outline our present knowledge of the AGB-star phenomenon is reviewed.
\end{abstract}

\section{AGB-stars}

Red giant stars have effectively divided themselves into two parts: a small, very hot $\left(\sim 10^{8} \mathrm{~cm}, \sim 10^{8} \mathrm{~K}\right)$ core that is strongly gravitationally bound, and a huge, much cooler mantle $\left(\sim 10^{13} \mathrm{~cm}, \sim 10^{3} \mathrm{~K}\right.$ at the surface $)$ where the external parts are only weakly gravitationally bound. We are here only interested in those red giants that have experienced central He-burning, and now populate the asymptotic giant branch (AGB). These are much rarer than those red giants that have only experienced central H-burning (the RGB-stars). The AGB-stars experience substantial mass loss from the surface, and huge circumstellar envelopes (CSEs) of gas and dust are formed with extents approaching $10^{19} \mathrm{~cm}$ and temperatures below $10 \mathrm{~K}$ in the external parts. This can be regarded as the third part of the star, and hence an AGB-star stretches more than 11 orders of magnitude in size and 7 orders of magnitude in temperature. The evolution of the star on the AGB depends on an intricate interplay between processes taking place in the three parts.

In this outline only the pure AGB-stars are considered, but it will set the stage also for the understanding of other fascinating aspects of the AGB-star phenomenon: binarity (Jorissen these proceedings, hereafter th.pr.), post-AGB objects (Van Winckel th.pr.), galactic structure (Dejonghe \& Van Caelenberg th.pr.), population studies (Lançon th.pr.), chemical evolution (Gustafsson \& Ryde 1998). A limited number of subjectively chosen references are given. More 
detailed reviews, including many of those in these proceedings, are mentioned in the text.

\section{The core}

The core consists of an electron-degenerate $\mathrm{C} / \mathrm{O}$-mixture [where the relative number of $\mathrm{C}$ and $\mathrm{O}$ depends on the still uncertain rate for the ${ }^{12} \mathrm{C}(\mathrm{n}, \gamma){ }^{16} \mathrm{O}$ reaction, $\mathrm{C} / \mathrm{O} \sim 0.1-1$ depending on mass], where no nuclear-burning processes take place (at the border towards supergiant stars electron-degenerate $\mathrm{O} / \mathrm{Ne}$ cores exist). It will eventually appear on the scene as a small, dense, hot (but gradually cooling) stellar remnant, a white dwarf (WD). In the classical models only stars with an initial mass less than $8-9 \mathrm{M}_{\odot}$ will have an electron-degenerate $\mathrm{C} / \mathrm{O}$-core (convective over-shooting may decrease this by $\sim 30 \%$, Bressan et al. 1993), and hence will not experience further nuclear burning processes, unless the core mass, $M_{\mathrm{c}}$, exceeds $1.4 \mathrm{M}_{\odot}$ and gravitational collapse ensues. Due to the short lifetime of an AGB-star $M_{\mathrm{c}}$ does not increase significantly during evolution $\left(\sim 10^{-7} \mathrm{M}_{\odot} \mathrm{yr}^{-1}\right)$, and the core mass of even a very massive AGB-star will not reach the $1.4 \mathrm{M}_{\odot}$-limit. [The fact that the descendants of AGB-stars, WDs, have sharply peaked mass distributions in the range $0.5-0.6$ supports a short AGBlifetime, and strongly indicates that substantial loss of mass must take place during the AGB (Weidemann 1990).] Only stars with a mass above $\sim 0.5 \mathrm{M}_{\odot}$ will enter the AGB following exhaustion of central He (Bressan et al. 1993), but only stars above $\sim 0.8 \mathrm{M}_{\odot}$ will reach the AGB within $\sim 14 \mathrm{Gyr}$. Thus, the AGB-stage is the final evolutionary stage for the large majority ( $>95 \%)$ of all stars in the Universe that have left the main sequence.

\section{The H-mantle}

Above the core lies a He-shell and above that a $\mathrm{H}$-mantle, and these are very active regions. He-burning provides the energy during the early evolution on the AGB (E-AGB). Eventually, $\mathrm{H}$ ignites at the base of the $\mathrm{H}$-mantle and from now on H-burning supplies the energy, except for very short periods $\left(<10^{3} \mathrm{yr}\right)$ of He-burning in the form of a thermo-nuclear runaway [He-shell flashes, or thermal pulses (TPs)] that repeats semi-periodically on time scales of $\sim 10^{4-5} \mathrm{yr}$ (longer the smaller the mass; Wagenhuber \& Groenewegen 1998). This defines the thermally pulsing AGB (TP-AGB; $~ 5-20 \%$ of the E-AGB time scale). The sudden increase in energy release during the TP extinguishes the H-burning and the outer convective parts of the $\mathrm{H}$-mantle may extend into the inter-shell region (enriched in ${ }^{12} \mathrm{C}$ ), leading to a dredge-up of material to the surface, the 3rd dredge-up [the process that produces C-stars; see Wallerstein \& Knapp 1998, for a review on this stellar type]. In the inter-shell region ${ }^{13} \mathrm{C}$ (still of uncertain origin) appears to be the neutron source, and a production of s-process elements will take place here (Lambert et al. 1995; Straniero et al. 1997). Finally, in the massive AGB-stars $\left(\geq 4 \mathrm{M}_{\odot}\right)$ the convective part of the $\mathrm{H}$-mantle may penetrate into the H-burning shell, leading to hot bottom burning (HBB; Boothroyd et al. 1993). HBB will effectively convert ${ }^{12} \mathrm{C}$ into ${ }^{13} \mathrm{C}$ and finally ${ }^{14} \mathrm{~N}$ (also ${ }^{7} \mathrm{Li}$ and ${ }^{26} \mathrm{Al}$ are produced, while e.g. ${ }^{19} \mathrm{~F}$ and ${ }^{18} \mathrm{O}$ are destroyed). 
The TP-induced dredge-up occurs easier in high- $M$ stars than in low- $M$ stars. Theoretically, for the moment, the lower mass limit to C-stars lies at $M_{\mathrm{MS}} \sim 1.5 \mathrm{M}_{\odot}$ for solar composition (Straniero et al. 1997). HBB prevents the more massive stars to become C-stars, and the upper mass limit to C-stars is $\sim 4 \mathrm{M}_{\odot}$. Since it must be easier to achieve $\mathrm{C}>\mathrm{O}$ in low- $Z$ stars we expect the relative number of $\mathrm{M}$ - and $\mathrm{C}$-stars to be a strong function of metallicity.

Our physical understanding of this region is good, but not perfect (Iben \& Renzini 1983; Frost \& Lattanzio 1996a; Blöcker th.pr., Lattanzio \& Forestini th.pr.). Two outstanding problems remain. First, the "crude" treatment of the convection using the mixing-length theory (the turbulence is local and instantaneous, characterized by one-size eddies, and a free parameter is introduced, the mixing length scale). Particular problems arise at the boundaries between convective and non-convective regions [the over-shoot problem (Frost \& Lattanzio $1996 \mathrm{~b})$, and the mixing]. During the TPs we have regions where the dynamical, thermal, and nuclear burning time scales are equal, and hence many common approximations become doubtful. When coupled to pulsation another, much shorter, time scale is introduced. Here we mention some important uncertainties. The dredge-up efficiency dependences on mass, metallicity, and time are crucial but poorly understood, e.g., some additional mixing may be required to produce low- $M$ C-stars (Herwig et al. 1997), or maybe degenerate TPs will do it (Frost et al. 1998b), and also the composition (the relative abundances of $\mathrm{He}, \mathrm{C}, \mathrm{O})$ of the dredged-up matter is uncertain. If mass loss decreases the $\mathrm{H}$ mantle mass, $M_{\mathrm{e}}$, sufficiently, $\mathrm{HBB}$ is quenched and high- $M$ stars may become luminous C-stars with low ${ }^{12} \mathrm{C} /{ }^{13} \mathrm{C}$-ratios (Frost et al. 1998a). There is also a possibility that $\mathrm{HBB}$ will produce luminous $\mathrm{M}$-stars with low ${ }^{12} \mathrm{C} /{ }^{13} \mathrm{C}$-ratios (e.g., evolved OH/IR-stars, Delfosse et al. 1997), and it may lead to stars that are highly enhanced in $\mathrm{N}$. The second major problem is the mass loss. A credible stellar evolution model must take this into account. The main problem is that it cannot yet be calculated from first principles, and hence some recipe that relates $\dot{M}$ to other stellar parameters must be used. Usually this results in substantially increasing mass loss with time, that is strongly modulated during the TPs, and final core masses that agree well with the mass-distribution of WDs (Blöcker 1995; Schröder et al. 1998). The AGB evolution is terminated when $M_{\mathrm{e}}$ decreases below a certain value, $\sim 0.001 \mathrm{M}_{\odot}$. This is almost certainly determined by the mass loss for all AGB-stars. Thus, we can expect a complex interplay between dredge-up, HBB, and mass loss that we still do not understand, and in the end the evolution of these giants depends on the details. We will not have reliable synthetic AGB evolution models before the main problems are solved (Groenewegen \& de Jong 1993; Marigo et al. 1996).

There is no full-proof direct observational evidence for the existence of TPs. Wood \& Zarro (1981) interpret period change rates as due to the behaviour during a TP. Olofsson et al. $(1996,1998 \mathrm{a})$ have suggested that the very geometrically thin gas-shells around a few C-stars are due to a recent TP. Sakurai's object could be a very recent born-again AGB-star, since drastic changes in elemental composition over short time scales (months) are apparent (Asplund et al. 1997; Asplund th.pr.).

The luminosity, $L$, of the star is determined by the rate of H-burning (except during a TP). There exists a linear relation (as long as the core ra- 
dius depends linearly on $M_{\mathrm{c}}$ ) between $L$ and $M_{\mathrm{c}}$ (which depends very little on other parameters, at least, after a few TPs, Wagenhuber \& Groenewegen 1998), $L \sim 57000\left(M_{\mathrm{c}}-0.5\right) \mathrm{L}_{\odot}$, and hence $L_{\max } \sim 56000 \mathrm{~L}_{\odot}\left(M_{\mathrm{bol}} \sim-7.1\right)$. However, it should always be remembered that the $L-M_{\mathrm{c}}$ relation applies only during a limited period between TPs ( $<50 \%$ of the time). Consequently, also an estimate of $M_{\mathrm{c}}$ using it may be wrong (by more than 10\%). HBB in the more massive stars will destroy the linear relation and produce higher $L$ than given by the $L-M_{\text {c }}$ relation (Boothroyd \& Sackman 1992).

At some point the H-mantle becomes unstable, and it will start to pulsate. There exists as yet no detailed model of the pulsation of AGB-stars, including e.g. a time-dependent treatment of convection (Tuchman th.pr.). The period calculations are sensitive to the outer boundary conditions (very difficult to define for a pulsating AGB-star). The growth rates are very sensitive to the details of the convection, as well as to the boundary conditions. It is not yet finally settled which stars pulsate in which mode. In principle, the problem requires only measurements of the stellar radius, $R$, and the period, $P$, and the use of the fundamental pulsation equation, $P=Q R^{3 / 2} M^{-1 / 2}$, where $Q$ is to be obtained from the model. However, the radius is not well defined in the case of an AGB-star, and it varies by as much as a factor of two with wavelength. The theoretical definition is usually in terms of the Rosseland mean optical depth. Taken at face values interferometric measurements of Miras at $\sim 0.8 \mu \mathrm{m}$ point to radii too large for fundamental-mode pulsation, if $M<1.5 \mathrm{M}_{\odot}$ (Haniff et al. 1995), while interferometer observations at $2.2 \mu \mathrm{m}$ are not so conclusive (van Belle et al. 1997; Feast th.pr.). However, there are strong arguments in favour of fundamental mode pulsation for Miras in the form of parallel K-log $P$ relations in the LMC (Wood \& Sebo 1996; Wood et al. th.pr.). The direct measurements may be reconciled with this if the measured radii are too large, e.g., due to scattering in the upper layers of the atmosphere.

\section{The atmosphere}

The outer part of the H-mantle is cool enough $(\leq 3500 \mathrm{~K})$ that formation of molecules will take place, and it is their presence that determines the spectral appearance of the stars, at least in the near-IR, and to some extent also the structure of their atmospheres (e.g., line blanketing). Our physical understanding of the atmosphere and the formation of the photospheric lines is good (Gustafsson 1989; Gustafsson \& Jørgensen 1994; Plez th.pr.). The "classical" sources of uncertainties are effective temperatures, surface gravities, sphericity, opacities, LTE analysis, and the approximate treatment of convection. The realization that the atmosphere is much more extended than previously thought and nonhydrostatic (systematic flows and shocks) is gradually introduced into models (see Sect. 5). Therefore, non-LTE effects are very likely present. Thermal instabilities, due to molecule and grain formation, may lead to inhomogeneities. Dust is possibly an important opacity source, and this requires a detailed knowledge of grain formation.

The determination of elemental abundances are far from trivial and elaborate models have been developed over the last decades. The spectra are extremely crowded, and usually a continuum level is difficult to find. Impressive 
results have nevertheless been obtained on elemental abundances (Smith \& Lambert 1990; Smith th.pr.), as well as on physical conditions [in the s-process region, Lambert et al. (1995), and in the HBB region, Smith et al. (1995)]. Atmosphere models also provide relations between important quantities like $L$ and $T_{\text {eff }}$ and broad-band colours (Bessel et al. 1998). Still, discrepancies between model and observations and, not so uncommonly, between different models applied to the same objects exist [e.g., Ohnaka \& Tsuji (1998) and references therein].

There are lots of crucial observations to be obtained here, such as very high spectral resolution data in the near-IR region for larger samples. Interferometer data at different wavelengths provide information on the stratification of the atmosphere, as well as on inhomogeneities at the surface. Evidence is gathering that the AGB-stars are asymmetric, but it is not clear whether the asymmetries are due to spots in the photospheres, clouds in the upper atmospheres, or to non-radial pulsations (Haniff et al. 1992; Karovska et al. 1997; Weigelt et al. 1996). An interesting effect of non-uniform disks is that the positions, parallaxes, and proper motions of AGB-stars measured by HIPPARCOS could have considerable uncertainties (e.g., $1000 \mathrm{R}_{\odot}$ at 50 and $250 \mathrm{pc}$ corresponds to 100 and 20 mas, respectively, considerably larger than the position resolution).

\section{The extended atmosphere}

The pulsation of the star will modulate the radiated power, but also deposit mechanical energy into the weakly gravitationally bound outer parts, the main effect being an atmosphere with a considerably increased scale height compared to a hydrostatic one. Shocks will develop, and at high enough altitudes grain condensation takes place in the post-shock gas. It appears that gas particles reach the escape velocity, under a broad range of conditions, due to the mechanical energy input augmented by radiation pressure on grains (and perhaps molecules).

Our physical understanding of this region is very limited (Höfner th.pr.). A complex interplay between different (non-linear) physical and chemical processes with different characteristic time scales takes place. In particular, grain formation is poorly understood in detail, but will have a significant effect on the thermal, hydrodynamical, and chemical structure of the upper atmosphere. The region is very likely inhomogeneous. Nevertheless, progress on dynamical stellar atmospheres is being made, in particular for C-stars where the grain formation is better understood (Fleischer et al. 1992; Höfner \& Dorfi 1997). Synthetic IR-light curves show complex (e.g., super- $P$ ) behaviours (Winters et al. 1994b), and the brightness distributions have considerable radial structure (Winters et al. 1995). Synthetic near-IR colour calculations have given results in accordance with observations (Alvarez \& Plez 1998). Also, synthetic spectra, in the $2-12 \mu \mathrm{m}$ range suitable for comparison with ISO-data, are being produced (Hron et al. 1998). The discrepancies are substantial and much remains to be done, but the prospects are excellent. Models with improved treatments of opacity show significant changes in the upper density structure (Höfner et al. 1998). Proper treatments of cooling in the post-shock gas and non-equilibrium processes are still lacking, and so is a proper coupling to a 'real' pulsating star. It is not clear to what extent the magnetic field is of importance for the structure and the 
dynamics (Hartquist \& Dyson 1997). Finally, we note the lack of similar models for M- and S-stars.

The importance of grain formation for the evolution of AGB-stars cannot be over-emphasized. It is therefore unfortunate that our knowledge of this complicated process is so rudimentary. The choice stands between a thermodynamic or a kinetic approach. In C-stars one possibility is that reactions between $\mathrm{C}_{2} \mathrm{H}_{2-}$ molecules eventually lead to soot particles (Cherchneff \& Cau th.pr.; Allain et al. 1997). However, many uncertainties remain, and other possible condensation seeds are SiC-, TiC-, and pure C-particles (Lodders \& Fegley th.pr.). In an O-rich environment $\mathrm{TiO}_{2}$ may be a possible nucleation species (Jeong et al. th.pr.). Also the radiative characteristics are uncertain (Henning th.pr.).

Crucial observations are provided by near- to mid-IR spectra, in particular of "simple" regions, and long time-series data. Detailed, multi-wavelength lightcurves provide important constraints (de Laverny et al. 1997). High-resolution observations provide information on atmosphere extension (Tuthill et al. 1995), dust formation zones (Danchi et al. 1994; Ivezić \& Elitzur 1996; Monnier th.pr.), inhomogeneities (Lopez et al. 1997; Weigelt et al. 1998), and departures from spherical symmetry (Lopez th.pr.). With new large telescopes we can look forward to detailed images of the extended atmospheres, including time variability. Multi-epoch images of $\mathrm{SiO}$ masers provide a very exciting view of the structure and dynamics of the upper atmospheric layers (Diamond \& Kemball th.pr.), and similar multi-epoch imaging of stellar $\mathrm{H}_{2} \mathrm{O}$ masers are also undertaken (Richards et al. th.pr.). There remains to determine whether we see matter or pattern motion. A recent very interesting development is the finding of a radio photosphere by Reid \& Menten (1997). A model places it at $\sim 2 R_{*}$, i.e., within the dust-condensation zone, where the density is $\sim 10^{12} \mathrm{~cm}^{-3}$ and the temperature $\sim 1600 \mathrm{~K}$. They detected no variations with time $(< \pm 15 \%)$, and this puts limits on the shocks passing through this region. Likewise, there may exist a quasi-static layer of molecular gas in the upper atmosphere (Tsuji et al. 1997).

A very important aspect of the dynamical atmosphere models is the possibility of deriving mass loss rates from first principles (Sedlmayr \& Dominik 1995; Fleischer et al. th.pr.). It appears that the levitation mechanism itself (shocks or possibly acoustic waves) can drive mass loss (and it may dominate for low $\dot{M}$ objects), but it is strongly augmented by the radiation pressure on dust, and possibly molecules, when this becomes effective. Models that give mass loss rates as a function of the fundamental stellar parameters have been presented for stationary (Winters et al. 1994a) and dynamical winds (Bowen \& Willson 1991; Arndt et al. 1997). However, Höfner et al (1998) have shown that more realistic opacities in the atmospheric models may result in (considerably) lower $\dot{M}$. We are still only in the beginning of obtaining reliable quantitative theoretical mass loss rates.

\section{The circumstellar envelope}

An AGB-star will eventually lose mass at a considerable rate in the form of a slow $\left(\sim 15 \mathrm{~km} \mathrm{~s}^{-1}\right)$ wind, and this forms a CSE of escaping gas- and dustparticles. The gas is to a large extent molecular with a composition that reflects the chemistry of the star. The molecules are located in envelopes of different 
sizes (the by far largest ones being those of $\mathrm{CO}$ and $\mathrm{H}_{2}$ ), or shells of different radii and widths, depending on their resistance to UV photons and the formation routes. The dust grains exist way beyond the molecular envelopes.

Our understanding of the CSE is relatively good (Habing 1996; Olofsson 1996; Bujarrabal th.pr.). However, the exact geometry of a CSE is uncertain, and the radial density distribution is determined by the (uncertain) mass loss history of the star, and it may be affected by the fact that one medium (dust) streams through another (gas), or a faster wind runs into a slower one. Furthermore, the details of the density and temperature structure of the gas is unknown. In the footsteps of the dynamical atmosphere models time-dependent hydrodynamical models that give the density, temperature, velocity, and chemical structures of CSEs are now being developed (Steffen et al. 1998, th.pr.).

Dust emission models have so far been based on stationary, smooth, spherical winds. The gross features of the spectral energy distributions can be well modelled using dirty amorphous silicates for O-rich CSEs (including the 10 and $18 \mu \mathrm{m}$ features; Le Sidaner \& Le Bertre 1996) and amorphous carbon for Crich CSEs (featureless; Groenewegen et al. 1998). Very important results are the tight $\dot{M}$-colour (in particular $\mathrm{K}-\mathrm{L}$ ) relations, although they differ for the two chemistries (Le Bertre \& Winters 1998). On the contrary the correlations between $\dot{M}$ and far-IR colours are poor. The more self-consistent models, i.e., those coupled to dynamical atmosphere models, have not yet been applied to large samples, but a detailed study of the C-star AFGL 3068 shows good agreement with a large data set (Winters et al. 1997).

The $11.3 \mu \mathrm{m}$ emission feature observed in C-rich CSEs can be well modelled if a small amount of $\mathrm{SiC}$ dust is added (Lorenz-Martins \& Lefèvre 1994). It seems that the discrepancy between AGB- and meteoritic-SiC grains has now been resolved. The presence of a $13 \mu \mathrm{m}$ emission feature in O-rich CSEs (Sloan et al. 1996) points to the existence of crystalline grains, possibly $\alpha-\mathrm{Al}_{2} \mathrm{O}_{3}$. Relatively narrow features beyond $20 \mu \mathrm{m}$ in O-rich CSEs also suggest the presence of crystalline material (its fraction appears to increase with $\dot{M}$ ) (Waters et al. 1996, th.pr.). The carriers of the $21 \mu \mathrm{m}$ feature in C-rich post-AGB CSEs (Kwok et al. 1995, th.pr.), and the $30 \mu \mathrm{m}$ feature in C-rich (AGB and postAGB) CSEs (Omont et al. 1995) have not yet been identified, although MgS may play a role for the latter (Szczerba et al. 1997). In very cold O-rich postAGB objects features, attributed to ice, appear at $\sim 43$ and $60 \mu \mathrm{m}$. There are post-AGB objects which show both O- and C-rich dust features (Waters th.pr.; Cohen et al. th.pr.). It is conceivable that in these objects there exists an O-rich (circumbinary) disk, and a C-rich AGB-CSE remnant.

Classification systems of dust features, that can be compared with different stellar populations, have been developed for the 8-14 $\mu \mathrm{m}$ range for O-rich (Hron et al. 1997) and C-rich CSEs (Sloan et al. 1998). These are interesting developments, but problems remain in the subtraction of the stellar- and dust-continuum.

Considerable progress has been done in our understanding of the origins of the molecules detected in CSEs (Glassgold 1996, th.pr.). The photospheric species are produced in a stellar atmosphere equilibrium chemistry, but the abundance varies with height in the atmosphere, and there may be effects due to shock waves, to chromospheric radiation, and to the formation of dust (Willacy 
\& Cherchneff 1998). Eventually, the photospheric species are photodissociated by the interstellar UV field [this requires a knowledge of the photodissociation properties of the molecule, the UV properties of the dust, and the properties (in the case of dissociation in lines, also the details) of the interstellar UV field]. The photodissociation will also initiate a circumstellar chemistry, where neutralneutral reactions appear to be the most effective (Millar \& Herbst 1994; Willacy $\&$ Millar 1997). In addition, formation on the surfaces of dust grains may be important for some species (e.g., $\mathrm{CH}_{4}, \mathrm{NH}_{3}$ ).

There are presently $\sim 60$ different molecular species detected in AGB-CSEs (Olofsson 1997). For the study of AGB stellar evolution the $\mathrm{OH}$ masers have been of utmost importance. There is a possibility that even more results will be derived from them, since several lines in the pump cycle of the $1612 \mathrm{MHz}$ line have now been detected (Justtanont et al. 1996; Sylvester et al. 1997). For the study of the properties of the CSEs, and the determination of reliable mass loss rates, the $\mathrm{CO}$ line emission has proven to be most important. The detections of highly excited $J$-lines from a number of molecular species by ISO will lead to significant improvements in our understanding of the inner regions of the CSE (Barlow th.pr.). The detailed testing of the circumstellar chemistry can in principle only be done for one object for the moment, IRC +10216 . To a first approximation the images clearly show the expected distribution of molecules with different origins (Guélin et al. 1996; Lucas \& Guélin th.pr.), but they also show spatial overlaps in emissions from species which from a chemical point of view are expected to be well separated (Guélin et al. 1993). This shows how important maps are for the interpretation.

A major problem in the interpretation of the molecular line data is the possibility of an inhomogeneous medium. Evidence for this comes from e.g. detailed light curves (Whitelock et al. 1997), high-resolution optical/IR images (Sect. 4), SiO maser maps (Boboltz et al. 1997), images of various molecular line emissions towards IRC+10216 (Guélin et al. 1996), and geometrically thin CO shells of a number of C-stars (Olofsson et al. 1996, 1998a). CO maps of evolved PNe indicate that already the AGB-CSE must have been inhomogeneous (Huggins et al. th.pr.). It seems reasonable to assume that the wind is already initially inhomogeneous at some level, but the further evolution of the density structure is not known.

\section{Properties of AGB-stars}

\subsection{Identification}

It is not an altogether simple task to isolate the AGB-stars within the much larger pool of red giants and other chemically peculiar stars. We can expect a major contamination with RGB-stars among the hardly variable and the low-luminosity AGB-stars, and a not negligible contamination with supergiants among the most luminous AGB-stars. There are ways (though not full-proof) to distinguish between these groups, e.g., JHK-diagrams, and comparison of CO radio line and $60 \mu \mathrm{m}$ fluxes (Josselin et al. 1998). There are also various ways of distinguishing between $\mathrm{M}$ - and C-stars if detailed spectra are not available, e.g., data from narrow filters centred on the $\mathrm{TiO}$ and $\mathrm{CN}$ absorption features, colour- 
colour diagrams (e.g., J-K vs [12]-[25]; Le Bertre et al. 1994), and circumstellar molecular radio line intensity ratios (Olofsson et al. 1998b).

There is also the more intricate problem of distinguishing between 'intrinsic' AGB-stars and 'extrinsic'-stars, i.e., stars with some AGB-characteristics due to mass overflow (Jorissen th.pr.). Here we find the 'extrinsic' S-stars which lie on the RGB (Van Eck et al. 1998), and the giant CH- (pop II) and Ba-stars (pop I). The dwarf C-stars probably also belongs to this category (maybe the dominant form of C-stars in the Galaxy). One should note here that many criteria for identifying 'extrinsic' stars do not work for extragalactic samples.

The J-stars are C-rich, but it has been speculated that they became Cenriched during or after the He-core flash (constitute $\sim 10 \%$ of the giant C-stars). In some cases (O-rich) matter, lost at the flash, may have been captured in a disk around a companion, hence forming a 'silicate C-star' (Lloyd Evans 1990). The R-type C-stars could be horizontal branch stars, for some reason C-enriched, and possibly the precursors of J-stars.

\subsection{Masses}

Distribution studies in the Galaxy suggest that the M-type semiregulars and intermediate-period $\left(300^{d}<P<400^{d}\right)$ Miras belong to the "thin disk" population and have progenitors in the $1.0-1.2 \mathrm{M}_{\odot}$ range (Jura \& Kleinmann $1992 \mathrm{a}, \mathrm{b}$; Kerschbaum \& Hron 1992). This is supported by kinematic studies, which also suggest that the mass increases with the period (Feast 1989). The short-period M-Miras $\left(P<300^{d}\right)$ belong to the "thick disk" population (Jura 1994). The obscured $\mathrm{OH} / \mathrm{IR}$-stars have on average higher- $M$ progenitors, in the range $2-3 \mathrm{M}_{\odot}$ (Blommaert et al. 1994; Wood et al. 1998). If the upper mass limit to Cstars is $\sim 4 \mathrm{M}_{\odot}$ we expect $\mathrm{OH} / \mathrm{IR}$-stars with $P>1000^{d}$ to be more massive than $4 \mathrm{M}_{\odot}$ since no C-stars with $P>800^{d}$ have been found, unless the long period is obtained through extensive mass loss (Habing 1996).

The C-star mass range appears narrower. Distribution and kinematic studies suggest that the progenitor masses may be somewhat higher, $1.2-1.6 \mathrm{M}_{\odot}$, than for M-stars of the same periodicity (Claussen et al. 1987; Guglielmo et al. 1998). An upper limit of $\sim 4 \mathrm{M}_{\odot}$ is consistent with the results from studies of obscured C-stars with high expansion velocity CSEs (Barnbaum et al. 1991; Kastner et al. 1993), but the exact limit is uncertain.

\subsection{Radii and effective temperatures}

Interferometer data suggest $R \sim 400-600 \mathrm{R}_{\odot}$ for Miras, with the non-Miras being on average $\sim 200 \mathrm{R}_{\odot}$ smaller (Haniff et al. 1995; van Belle et al. 1997). These results are still dependent on the uncertain distances, and the models, since the angular sizes have been converted to photospheric sizes. For the effective temperature van Belle et al. find on average $T_{\text {eff }} \sim 2200-2700 \mathrm{~K}$ for Miras, with the non-Miras being on average $\sim 600 \mathrm{~K}$ warmer in all groups (the spread in each group is considerable; see also Perrin et al. 1998). van Belle et al. (1996) also find that $T_{\text {eff }}$ change with phase in the expected manner, and that $R$ decreases linearly with increasing $T_{\text {eff }}$, and Burns et al. (1998) have detected periodic size variations for the M-Mira R Leo. 


\subsection{Luminosities}

It has been long realized that in the optical surveys of the LMC there is a lack of C-stars brighter than $M_{\text {bol }} \sim-6$, and in general a deficit of AGB-stars brighter than this (Frogel \& Blanco 1990; Hughes \& Wood 1990; Costa \& Frogel 1996). HBB and mass loss can explain this, at least qualitatively. However, the possibility exists that high- $L$ sources can be found among the obscured objects missed by optical surveys. For instance, in the Galaxy the most luminous objects are the very long- $P$ OH/IR-stars, $M_{\text {bol, } \max } \sim-7$ to -7.5 and $1000^{d}<P<2800^{d}$ (van Langevelde et al. 1990). The preliminary analysis of a small sample of IRAS sources in the LMC indicate that the luminosity function for these objects peaks at $M_{\text {bol }} \sim-5.8$, compared to $\sim-4.9$ in the optical surveys, and there are at least three C-stars brighter than $M_{\text {bol }}=-6.0$ (van Loon et al. 1998; Groenewegen \& Blommaert 1998). On the other side of the coin, we have the faint C-stars $\left(M_{\text {bol }} \leq-1.8\right)$ detected in the SMC (Westerlund 1995; could they be 'extrinsic' C-stars?).

The fundamental pulsation equation provides a relation between $L$ and $P$ (Sect. 3), which is observationally confirmed in the form of relatively tight $P$ $L$ relations up to $\sim 500^{d}$ (Groenewegen \& Whitelock 1996). For longer periods there are significant deviations (Reid et al. 1995; Zijlstra et al. 1996; Blommaert et al. 1998; Wood et al. 1998). The effects of metallicity are uncertain, but probably small, although Wood et al. (1998) argue that a metallicity effect may be present in a Galactic Centre sample of $\mathrm{OH} / \mathrm{IR}$-stars. In the same sample the long- $P$ objects lie distinctly below the predicted $L$, which could be an $\dot{M}$ effect: substantial mass loss moves the star to longer periods with little change in luminosity. It seems reasonable that the $P-L$ relation mainly reflects a mass sequence, i.e., evolutionary effects are small.

\subsection{Elemental abundances}

Smith \& Lambert (1990) present results for the CNO-abundances of M-, MS-, $\mathrm{S}-, \mathrm{SC}$-, and C-stars, the average C/O-ratios are $0.41(\mathrm{M}), 0.49(\mathrm{MS}), 0.57(\mathrm{~S})$, 0.95 (SC), and 1.15 (C) with some considerable scatter within each group. The $\mathrm{s}$-processes elements also show a clear increase as the $\mathrm{C} / \mathrm{O}$-ratio increases in the $\mathrm{M}$ to $\mathrm{C}$ sequence. Some peculiarities remain and may indicate systematic problems in the analysis, e.g., the markedly higher abundance of $\mathrm{N}$ in MS- and S-stars compared to the M- and C-stars, and the low C/O-ratio in C-stars (some $\mathrm{PNe}$ have $\mathrm{C} / \mathrm{O}$ well above 1.5 and up to $\sim 4)$. The next step here is definitely to get the same type of data, e.g., on CNO-abundances, for the stars in the Magellanic Clouds.

A most beautiful finding is that information about the elemental abundances of AGB-stars lies at our doorstep, enclosed in the meteorites (Zinner 1998). These contain $\mathrm{Al}_{2} \mathrm{O}_{3}$ and $\mathrm{SiC}$ grains, which carry information about $\mathrm{O}$ isotopes, ${ }^{26} \mathrm{Al},{ }^{12} \mathrm{C} /{ }^{13} \mathrm{C},{ }^{14} \mathrm{~N} /{ }^{15} \mathrm{~N}$, and a large number of s-process elements. In particular, isotope patterns can be determined to an impressive accuracy (Zinner \& Amari th.pr.). 


\subsection{Space densities and galactic distribution}

Groenewegen et al. (1995b) estimate that in the solar neighbourhood the relative numbers of $\mathrm{M}-$-, $\mathrm{S}$-, and $\mathrm{C}$-stars are $\mathrm{C} / \mathrm{M} \sim 0.2$ and $\mathrm{S} / \mathrm{C} \sim 0.3$ for stars with $P<400^{d}$, but for the rarer, more obscured objects the C/M-ratio is more like 0.5 (Jura \& Kleinmann 1989). In the LMC the C/M-ratio is in the range 0.6-2.2 for optically visible AGB-stars (Hughes \& Wood 1990), while for the obscured objects it lies in the range $0.2-0.8$ and decreases with luminosity (van Loon et al. 1998).

The radial distributions of $\mathrm{M}$ - and C-stars are very different in the Galaxy. The surface density of 'thin disk' M-stars follow an exponential disk with a scale length of $\sim 4 \mathrm{kpc}$ (Habing 1988; Blommaert et al. 1993). The C-star distribution is essentially constant within $\sim 3 \mathrm{kpc}$ of the Sun (Jura \& Kleinmann 1990; Guglielmo et al. 1998). Thus, there is a marked trend in the C/M-ratio with galactocentric distance, very likely due to the effects of metallicity. Lloyd Evans (1984) showed that in the LMC the luminosity where C-stars become abundant increases with decreasing cluster age (hence increasing metallicity). The same metallicity effect is present in M31 (Brewer 1996).

\subsection{Mass loss}

There remains no doubt that the AGB-evolution is accompanied by substantial mass loss (observationally detectable when it exceeds $\sim 10^{-8} \mathrm{M}_{\odot} \mathrm{yr}^{-1}$ ). However, when it comes to the details like $\dot{M}\left(t, M_{\mathrm{MS}}, Z, \theta, \phi\right)$ and dependences on pulsational behaviour things become more difficult. In the following discussion one should bear in mind that these dependences are not easily separated.

For the short-term behaviour we have information at different time scales: a density modulation in the CSE of the M-Mira IK Tau corresponds to a time scale of $\sim 10 \mathrm{yr}$ (Hale et al. 1997), $\mathrm{CO}$ emission from extreme OH/IR-stars suggests considerably increased mass loss over the last $\sim 10^{3} \mathrm{yr}$ (Delfosse et al. 1997), extended $1.3 \mathrm{~mm}$ emission from IRC+10216 indicates several periods of enhanced mass loss during the last $10^{3} \mathrm{yr}$ (Groenewegen et al. 1997), detached $\mathrm{CO}$ and dust shells around C-stars suggest episodic mass loss in the range $(1-20) \times 10^{3} \mathrm{yr}$ (Olofsson et al. 1996; Izumiura et al. 1997), extended $60 \mu \mathrm{m}$ emission also indicates time variations (for some reason the semiregulars dominate among the extended sources; Young et al. 1993), and the HST-image of the Egg nebula shows a large number of concentric rings, interpreted as a mass loss rate modulation occuring every 150-450yr during the final AGB evolution (Sahai et al. 1998b).

For the long-term behaviour we are mainly restricted to statistical studies. Habing (1996) suggests a time dependence since there are optical Miras and obscured $\mathrm{OH} / \mathrm{IR}$-stars with the same $L$, i.e., for a given $L$ (and hence $M$ ) stars may have different $\dot{M}$, and among AGB-stars with the same $L$, but with different (long) $P$, the longer- $P$ stars tend to have the highest $\dot{M}$ (assuming of course that the longer $P$ is attributed to $\dot{M}$ ). On the other hand, correlations between $\dot{M}$ and $P$ indicate an $M$-dependence. The tight $P-L$ relations for Miras suggest that very intense mass loss, that significantly alters $M$ and hence $P$, must be of relatively short duration. Thus, it appears that on average $\dot{M}$ increases with time, but that the achievable maximum mass loss rate increases with $M_{\mathrm{MS}}$. 
We do not know when the onset of mass loss occurs. It is likely that we will find the information among the low $\dot{M}\left(<10^{-7} \mathrm{M}_{\odot} \mathrm{yr}^{-1}\right)$ objects (Kerschbaum \& Olofsson 1998). At the other end, it is clear that the mass loss drops by orders of magnitude when the star leaves the AGB. However, the details are unknown, and there are considerable uncertainties in the interpretation (Meixner et al. 1997; Steffen et al. 1998; Schröder et al. 1998).

The mass loss characteristics dependence on metallicity mainly stems from studies of $\mathrm{OH} / \mathrm{IR}$-stars in the Galactic Centre, the Bulge, the anti-centre and in the LMC. It appears from these that both $\dot{M}$ and the gas expansion velocity increase (weakly) with metallicity (Zijlstra et al. 1996; Blommaert et al. 1998; Wood et al. 1998). Groenewegen et al. (1995a) studied three stars (in the Galaxy, LMC, and SMC) with similar $P$ and $L$ and derived CSE dust optical depths ratios of $\sim 15: 10: 1$, very likely a metallicity effect.

The geometry of the mass loss is another important property for understanding the mass loss mechanism. The conclusion drawn from observations of sufficiently high sensitivity and spatial resolution is that the AGB mass loss is essentially isotropic (Bowers \& Johnston 1990; Neri et al. 1998; Olofsson et al. 1996, 1998a; Groenewegen et al. 1997), though there are exceptions among the low $\dot{M}$ sources where examples of bipolar outflows have been found (Kahane \& Jura 1996; Kahane et al. 1996). This picture of over-all spherical symmetry contrasts with the appearance of post-AGB objects where clear axi-symmetry is the rule (Alcolea et al. 1996; Bujarrabal et al. 1998; Sahai et al. 1998a). A current acceptable picture is one of isotropic mass loss on the AGB, except perhaps at the final stages. During post-AGB evolution a high-velocity wind, maybe inherently bipolar or channelled by a (weak) equatorial density enhancement in the AGB envelope, will effectively lead to an axi-symmetric CSE, where the brightness contrast may be much higher than the total density contrast. In some objects strong equatorial density enhancements in the form of disks (of unknown origin) seem to be present.

\section{Prospects}

The prospects for progress are very good. On the theoretical side the computers become increasingly faster, but this is partly counteracted by the realization that the models must include a multitude of complex physical and chemical phenomena, and the very likely importance of significant departures from spherical symmetry. On the observational side sensitive high spectral resolution observations, high spatial resolution observations (in all wavelength regimes), and the sensitive surveys performed (e.g., DENIS, 2MASS, and by-products of projects like MACHO; Epchtein th.pr.; Loup et al. th.pr.; Wood et al. th.pr.) will significantly increase our knowledge of the behaviour of AGB-stars. In particular, the effects of metallicity (Groenewegen th.pr.; Zijlstra th.pr.) will be clarified. It is important in this context to remember that AGB-stars in the LMC/SMC were close to the detection limit for IRAS, meaning that there is far from a complete inventory of the mass-losing AGB populations in these objects. It is also very important that the effects of binarity are sorted out (Iben 1991; Jorissen th.pr.), in particular, when interpreting results from external galaxies. 
Acknowledgments. I am grateful to the Swedish Natural Science Research Council (NFR) and the French Foreign Office for travel support.

\section{References}

Alcolea J., Bujarrabal V., Sánchez Contreras C., 1996, A\&A 312, 560

Allain T., Sedlmayr E., Leach S., 1997, A\&A 323, 163

Alvarez R., Plez B., 1998, A\&A 330, 1109

Arndt T.U., Fleischer A.J., Sedlmayr E., 1997, A\&A 327, 614

Asplund M., Gustafsson B., Lambert D.L., Rao N.K., 1997, A\&A 318, 521

Barnbaum C., Morris M., Zuckerman B., 1991, AJ 102, 289

Bessel M.S., Castelli F., Plez B., 1998, A\&A 333, 231

Blommaert J.A.D.L., van der Veen W.E.C.J., Habing H.J., 1993, A\&A 267, 39

Blommaert J.A.D.L., van der Veen W.E.C.J., Van Langevelde H.J., Habing H.J., Sjouwerman L.O., 1998, A\&A 329, 991

Blommaert J.A.D.L., Van Langevelde H.J., Michiels W.F.P., 1994, A\&A 287, 479

Blöcker T., 1995, A\&A 297, 727

Boboltz D.A., Diamond P.J., Kemball A.J., 1997, ApJ 487 L147

Boothroyd A.I., Sackmann I.-J., 1992, ApJ 393, L21

Boothroyd A.I., Sackmann I.-J., Ahern S.C., 1993, ApJ 416, 762

Bowen G.H., Willson L.A., 1991, ApJ 375, L53

Bowers P.F., Johnston K.J., 1990, ApJ 354, 676

Bressan A., Fagotto F., Bertelli G., Chiosi C., 1993, A\&AS 100, 647

Brewer J.P., 1996, PASP 108, 379

Bujarrabal V., Alcolea J., Neri R., 1998, ApJ 504, 915

Burns D., Baldwin J.E., Boysen R.C., et al., 1998, MNRAS 297, 462

Claussen M.J., Kleinmann S.G., Joyce R.R., Jura M., 1987, ApJS 65, 385

Costa E., Frogel J.A., 1996, AJ 112, 2607

Danchi W.C., Bester M., Degiacomi C.G., Greenhill L.J., Townes C.H., 1994, AJ 107,1469

Delfosse X., Kahane C., Forveille T., 1997, A\&A 320, 249

de Laverny P., Geoffray H., Jorda L., Kopp M., 1997, A\&AS 122, 415

Feast M.W., 1989, in Evolution of Peculiar Red Giant Stars, H.R. Johnson \& B. Zuckerman (eds.), IAU Coll. 106, Cambridge University Press, Cambridge, p. 26

Fleischer A.J., Gauger A., Sedlmayr E., 1992, A\&A 266, 321

Frogel J.A., Mould J., Blanco V.M., 1990, ApJ 352, 96

Frost C.A., Cannon R.C., Lattanzio J.C., Wood P.R., Forestini M., 1998a, A\&A 332, L17

Frost C.A., Lattanzio J.C., Wood P.R., 1998b, ApJ 500, L355

Frost C.A., Lattanzio J.C., 1996a, in Stellar Evolution: What Should Be Done?, A. Noels et al. (eds.), Proc. of the 32nd Liege Coll., p. 307 
Frost C.A., Lattanzio J.C., 1996b, ApJ 473, 383

Glassgold A.E., 1996, ARA\&A 34, 241

Groenewegen M.A.T., Blommaert J.A.D.L., 1998, A\&A 332, 25

Groenewegen M.A.T., de Jong T., 1993, A\&A 267, 410

Groenewegen M.A.T., van der Veen W.E.C.J., Lefloch B., Omont A., 1997, A\&A 322, L21

Groenewegen M.A.T., Whitelock P.A., 1996, MNRAS 281, 1347

Groenewegen M.A.T., Whitelock P.A., Smith C.H., Kerschbaum F., 1998, MNRAS 293, 18

Groenewegen M.A.T., Smith C.H., Wood P.R., Omont A., Fujiyoshi T., 1995a, ApJ 449, L119

Groenewegen M.A.T., van den Hoeck L.B., de Jong T., 1995b, A\&A 293, 381

Guélin M., Lucas R., Cernicharo J., 1993, A\&A 280, L19

Guélin M., Lucas R., Neri R., 1996, in Science with Large Millimetre Arrays, P.A. Shaver (ed.), Springer, Berlin, p. 276

Guglielmo F., Le Bertre T., Epchtein N., 1998, A\&A 334, 609

Gustafsson B., 1989, ARA\&A 27, 701

Gustafsson B., Jørgensen U.G., 1994, A\&AR 6, 19

Gustafsson B., Ryde N., 1998, in The Carbon Star Phenomenon, R. Wing (ed.), IAU Symp. 177, Kluwer, Dordrecht, in press

Habing H.J., 1988, A\&A 200, 40

Habing H.J., 1996, A\&AR 7, 97

Hale D.D.S., Bester M., Danchi W.C., et al., 1997, ApJ 490, 407

Haniff C.A., Scholz M., Tuthill P.G., 1995, MNRAS 276, 640

Haniff C.A., Ghez A.M., Gorham P.W., Kulkarni S.R., Matthews K., Neugebauer G., 1992, AJ 103, 1662

Hartquist T.W., Dyson J.E., 1997, A\&A 319, 589

Herwig F., Blöcker T., Schönberner D., El Eid M., 1997, A\&A 324, L81

Hron J., Aringer B., Kerschbaum F., 1997, A\&A 322, 280

Hron J., Loidl R., Höfner S., Jørgensen U.G., Aringer B., Kerschbaum F., 1998, A\&A 335, L69

Hughes S.M.G., Wood P.R., 1990, AJ 99, 784

Höfner S., Dorfi E.A., 1997, A\&A 319, 648

Höfner S., Jørgensen U.G., Loidl R., Aringer B., 1998, A\&A 340, 497

Iben I., Jr., 1991, ApJS 76, 55

Iben I., Jr., Renzini A., 1983, ARA\&A 21, 271

Ivezić Ž., Elitzur M., 1996, MNRAS 279, 1019

Izumiura H., Waters L.B.F.M., de Jong T., Loup C., Bontekoe Tj.R., Kester D.J.M., 1997, A\&A 323, 449

Josselin E., Loup C., Omont A., Barnbaum C., Nyman L.-A., Sèvre F., 1998, A\&AS 129,45

Jura M., 1994, ApJ 422, 102 
Jura M., Kleinmann S.G., 1989, ApJ 341, 359

Jura M., Kleinmann S.G., 1990, ApJ 364, 663

Jura M., Kleinmann S.G., 1992a, ApJS 79, 105

Jura M., Kleinmann S.G., 1992b, ApJS 83, 329

Justtanont K., de Jong T., Helmich F.P., et al., 1996, A\&A 315, L217

Kahane C., Audinos P., Barnbaum C., Morris M., 1996, A\&A 314, 871

Kahane C., Jura M., 1996, A\&A 310, 952

Karovska M., Hack W., Raymond J., Guinan E., 1997, ApJ 482, L175

Kastner J., Forveille T., Zuckerman B., Omont A., 1993, A\&A 275, 163

Kerschbaum F., Hron J., 1992, A\&A 263, 97

Kerschbaum F., Olofsson H., 1998, A\&A 336, 654

Kwok S., Hrivnak B.J., Geballe Th.R., 1995, ApJ 454, 394

Lambert D.L., Smith V.V., Busso M., Gallino R., Straniero O., 1995, ApJ 450, 302

Le Bertre T., Epchtein N., Guglielmo F., Le Sidaner P., 1994, Ap\&SS 217, 105

Le Bertre T., Winters J.M., 1998, A\&A 334, 173

Le Sidaner P., Le Bertre T., 1996, A\&A 314, 896

Lloyd Evans T., 1984, MNRAS 208, 447

Lloyd Evans T., 1990, MNRAS 243, 336

Lopez B., Danchi W.C., Bester M., et al., 1997, ApJ 488, 807

Lorenz-Martins S., Lefèvre J., 1994, A\&A 291, 831

Marigo P., Bressan A., Chiosi C., 1996, A\&A 313, 545

Meixner M., Skinner C.J., Graham J.R., Keto E., Jernigan J.G., Arens J.F., 1997, ApJ 482, 897

Millar T.J., Herbst E., 1994, A\&A 288, 561

Neri R., Kahane C., Lucas R., Bujarrabal V., Loup C., 1998, A\&AS 130, 1

Ohnaka K., Tsuji T., 1998, A\&A 335, 1018

Omont A., Moseley H.S., Cox P., et al., 1995, ApJ 454, 819

Olofsson H., 1996, Ap\&SS 245, 169

Olofsson H., 1997, in Molecular Astrophysics: Probes \& Processes, E. van Dishoeck (ed.), IAU Symp. No. 178, Kluwer, Dordrecht, p. 457

Olofsson H., Bergman P., Eriksson K., Gustafsson B., 1996, A\&A 311, 587

Olofsson H., Bergman P., Lucas R., Eriksson K., Gustafsson B., Bieging J.H., 1998a, A\&A 330, L1

Olofsson H., Lindqvist M., Nyman L.-A., Winnberg A., 1998b, A\&A 329, 1059

Perrin G., Coudé du Foresto V., Ridgway S.T., et al., 1998, A\&A 331, 619

Reid M.J., Menten K.M., 1997, ApJ 476, 327

Reid I.N., Hughes S.M.G., Glass I.S., 1995, MNRAS 275, 331

Sahai R., Hines D.C., Kastner J.H., et al., 1998a, ApJ 492, L163

Sahai R., Trauger J.T., Watson A.M., et al., 1998b, ApJ 493, 301

Schröder K.-P., Winters J.M., Sedlmayr E., 1998, A\&A in press 
Sedlmayr E., Dominik C., 1995, Sp. Sc. Rev. 73, 211

Sloan G.C., Le Van P.D., Little-Marenin I.R., 1996, ApJ 463, 310

Sloan G.C., Little-Marenin I.R., Price S.D., 1998, AJ 115, 809

Smith V.V., Lambert D.L., 1990, ApJS 72, 387

Smith V.V., Plez B., Lambert D.L., Lubowich D., 1995, ApJ 441, 735

Steffen M., Szczerba R., Schönberner D., 1998, A\&A 337, 149

Straniero O., Chieffi A., Limongi M., Busso M., Gallino R., Arlandini C., 1997, ApJ 478, 332

Sylvester R.J., Barlow M.J., Nguyen-Q-Rieu, et al., 1997, MNRAS 291, L42

Szczerba R., Omont A., Volk K., Cox P., Kwok S., 1997, A\&A 317, 859

Tsuji T., Ohnaka K., Aoki W., Yamamura I., 1997, A\&A 320, L1

Tuthill P.G., Haniff C.A., Baldwin J.E., 1995, MNRAS 277, 1541

van Belle G.T., Dyck H.M., Benson J.A., Lacasse M.G., 1996, AJ 112, 2147

van Belle G.T., Dyck H.M., Thompson R.R., Benson J.A., Kannappan S.J., 1997, AJ 114, 2150

Van Eck S., Jorissen A., Udry S., Mayor M., Pernier B., 1996, A\&A 329, 971

van Langevelde H.J., van der Heiden R., van Schooneveld C., 1990, A\&A 239, 193

van Loon J.Th., Zijlstra A.A., Whitelock P.A., et al., 1998, A\&A 329, 169

Wagenhuber J., Groenewegen M.A.T., 1998, A\&A 340, 183

Wallerstein G., Knapp G.R., 1998, ARA\&A 36, 369

Waters L.B.F.M., Molster F.J., de Jong T., et al., 1996, A\&A 315, L361

Weidemann V., 1990, ARA\&A 28, 103

Weigelt G., Balega Y., Blöcker T., Fleischer A.J., Osterbart R., Winters J.M., 1998, A\&A 333, L51

Weigelt G., Balega Y., Hofmann K.-H., Scholz M., 1996, A\&A 316, L21

Westerlund B.E., Azzopardi M., Breysacher J., Rebeirot E., 1995, A\&A 303, 107

Whitelock P, Feast M., Marang F., Overbeek M.D., 1997, MNRAS 288, 512

Willacy K., Cherchneff I., 1998, A\&A 330, 676

Willacy K., Millar T.J., 1997, A\&A 324, 237

Winters J.M., Dominik C., Sedlmayr E., 1994a, A\&A 288, 255

Winters J.M., Fleischer A.J., Gauger A., Sedlmayr E., 1994b, A\&A 290, 623

Winters J.M., Fleischer A.J., Gauger A., Sedlmayr E., 1995, A\&A 302, 483

Winters J.M., Fleischer A.J., Le Bertre T., Sedlmayr E., 1997, A\&A 326, 305

Wood P.R., Habing H.J., McGregor P.J., 1998, A\&A 336, 925

Wood P.R., Sebo K.M., 1996, MNRAS 282, 958

Wood P.R., Zarro D.M., 1981, ApJ 247, 247

Young K., Phillips T.G., Knapp G.R., 1993, ApJ 409, 725

Zijlstra A.A., Loup C., Waters L.B.F.M, Whitelock P.A., van Loon J.Th., Guglielmo F., 1996, MNRAS 279, 32

Zinner E., 1998, ARE\&PS 26, 147 